\title{
Estudo comparativo sobre burnout em professores de ensino regular e educação especial
}

\section{Burnout: A comparative study between teachers (non-specialists and specialist in SEN)}

\author{
Adelinda Candeias, Inês Calisto, Liberata Borralho \\ Universidade de Évora
}

\begin{abstract}
Resumo
O objetivo do estudo é comparar os níveis de burnout em professores do ensino regular e educação especial em escolas públicas portuguesas que trabalham com alunos com Necessidades Educativas Especiais.

A amostra é constituída por 7058 docentes de ensino regular e 451 de educação especial. O estudo dos resultados baseou-se numa análise de variância univariada. É o grupo de ensino regular que revela maiores níveis de realização pessoal e profissional e despersonalização. Neste grupo, são as mulheres que estão mais exaustas emocionalmente e os homens mais despersonalizados, sugerindo-se para este grupo a aplicação de um programa de prevenção de burnout.

Palavras-chave: escolas públicas; professores; burnout; ensino regular; educação especial.
\end{abstract}

\begin{abstract}
The aim of the study is to compare the levels of burnout in teachers of regular education and special education in Portuguese public schools that work with students with disabilities. The sample is composed of 7058 regular teaching staff and 451 special education teachers. The study of the results was based on a univariate analysis of variance. It is the regular teaching group that reveals higher levels of personal and professional achievement and depersonalization. In this group, it is the women who are more emotionally exhausted and the men more depersonalized, suggesting to this group the application of a program of prevention of burnout.

Keywords: public schools; teachers; burnout; regular education; special education.
\end{abstract}

\section{Introdução}

A escola pode constituir um contexto de stresse dadas as elevadas exigências que lhe são intrínsecas, associadas à responsabilidade e à exigência de educar as crianças e jovens. $\mathrm{O}$ stresse, quando prolongado no tempo, dá origem ao burnout, resultando de um processo de longa duração, em que o trabalhador sente que os recursos para lidar com as exigências estão esgotados (Santos, 2011). Carlotto (2002) descreve o burnout em professores como um fenómeno complexo e multidimensional, resultante da interação entre aspetos individuais e o ambiente de trabalho.

A proposta de inclusão educativa criada pelo Decreto-lei 3/2008, desencadeou profundas mudanças na forma de compreender e estruturar o trabalho dos professores Educação Escolar e Pré-escolar (Gomes, 2010). Na escola de natureza inclusiva, os professores enfrentam situações que implicam mudanças nas suas práticas educativas que permitam atender à diversidade e heterogeneidade dos alunos e simultaneamente garantir a igualdade de oportunidades e os apoios necessários que permitam o sucesso de todos e a qualidade de vida e bem-estar de todos os alunos (Baptista, 2013).

A inclusão, em termos educativos, faz mais sentido se for perspetivada como educação inclusiva, o que significa que a escola, para além de proporcionar aos alunos um espaço comum, deve proporcionar-lhes, também, oportunidades para que façam aprendizagens significativas, no entanto, embora se aceite facilmente que a escola é um lugar que proporciona a interação de aprendizagens significativas a todos os seus alunos, não é fácil geri-las, em particular quando alguns alunos têm problemáticas complexas ou quando os recursos são insuficientes (Silva, 2011).

Neste contexto há um conjunto de mudanças que são requeridas em termos de atitude face à inclusão de alunos com NEE, práticas pedagógicas diferenciadas, organização e à gestão da escola. A inclusão de crianças com NEE implica a preparação por parte dos docentes e a necessidade de existência de profissionais especializados, para que possam contribuir para a diminuição da exclusão escolar, para uma melhoria do rendimento dos alunos e para que possam servir de suporte, tanto para estas crianças e jovens, como também para os professores em geral, contudo, estas medidas acabam por trazer aos profissionais grandes dificuldades (Capitão, 2010). 
De forma global, tanto os professores de ensino regular como os de educação especial apoiam a inclusão educativa e acreditam que os alunos com dificuldades de aprendizagens e outras incapacidades ligeiras ou moderadas, têm direito a uma educação em sala de aula regular, porém existe alguma discordância relativamente à integração dos alunos em tempo inteiro em contexto sala de aula (Baptista, 2013). Alguns estudos revelam que os professores, na sua maioria, têm atitudes positivas perante a inclusão (Avramidis, Bayliss \& Burden, 2000; Avramidis \& Kalyva, 2007; Jerlinder, Danermark \& Gill, 2010; Memisevic \& Hodzic, 2011; Pinto \& Morgado, 2012), contudo, são também mencionadas algumas condicionantes, as quais podem ser um entrave para as boas práticas inclusivas por parte destes profissionais (Pinto \& Morgado, 2012).

Relativamente às dificuldades apresentadas pelos professores, alguns dos estudos realizados nesta área (por exemplo, Silva, 2011 e Cruz, 2012) demonstram existirem dificuldades como a falta de coordenação dos horários entre os professores de ensino regular e os professores de educação especial, o elevado número de horas de trabalho burocrático e por vezes, o número elevado de alunos com NEE que são acompanhados no trabalho conjunto entre os professores do ensino regular e os professores de educação especial. A falta de coordenação na criação de horários dos diferentes professores, que lhes permitam o envolvimento estruturas de colaboração tem sido também um obstáculo apontado.

No que concerne ao trabalho dos professores de educação especial, as suas funções são mais abrangentes do que as de um professor de ensino regular, uma vez que trabalha com crianças e adolescentes com NEE, cabe-lhe fazer uma adaptação ao mesmo e prever a utilização de apoios compensatórios, face às dificuldades detetadas. Neste sentido, terá que incluir uma certa inovação nas estratégias e métodos de ensino e fazer uma adaptação individualizada do ensino e diferenciação pedagógica, procedendo à mudança de conteúdos escolares, com o objetivo de melhorar e enriquecer o currículo escolar e melhorar o nível de realização do aluno Em relação ao trabalho em articulação, existem alguns constrangimentos por parte dos professores de ensino regular, estando estes relacionados com o processo de mudança, uma vez que estes profissionais sentem não possuir a formação necessária e adequada para trabalhar em sala de aula regular com alunos com NEE, dado que não dominam os problemas específicos (Cruz, 2012).

A tarefa de ensinar por si só é complexa, uma vez que para além deste ato, é necessária competência profissional, reflexão sobre as práticas, ser-se tolerante e respeitar a individualidade. Quando o professor se depara com os alunos que têm dificuldades de aprendizagem, apresentando algumas limitações que dificultam o seu processo de aprendizagem, o trabalho do professor torna-se mais complexo, podendo fazer com que este duvide da sua capacidade profissional, despoletando sentimentos de tristeza e impotência para resolver as situações (Barasuol, 2004). Assim, também a experiência que cada professor tem em trabalhar com crianças com NEE, pode influenciar as atitudes relativamente à inclusão (Pinto \& Morgado, 2012). De acordo com alguns estudos realizados, os professores que têm mais experiência com estas crianças demonstram atitudes mais positivas perante a inclusão (Avramidis, Bayliss \& Burden, 2000; Čagran \& Schmidt, 2011; de Boer, Pijl \& Minnaert, 2010; Gyimah, Sugden \& Pearson, 2009; Jerlinder, Danermark \& Gill, 2010; Rakap \& Kaczmarek, 2010; Pinto \& Morgado, 2012).

\section{Burnout na docência}

A docência é uma das profissões que apresenta níveis mais elevados de stresse e burnout (Carlotto, 2002; Martins, 2008). Uma das possíveis consequências do stresse (distress) prolongado ou crónico na profissão docente é a síndrome de burnout, sendo este utilizado para descrever o ciclo degenerativo da eficácia docente (Romão, 2012). A síndrome de burnout parece ser determinada pela utilização de estratégias de confronto não adaptativas, levando a um esgotamento dos recursos emocionais. De acordo com Maslach, Schaufeli e Leiter (2001) o burnout caracteriza-se de acordo com três dimensões: esgotamento emocional, despersonalização, entendida como distanciamento ou cinismo, e falta de realização pessoal, com sentimentos de incompetência e fracasso.

De acordo com Facion (Morais, 2011), uma das consequências do burnout em professores inseridos no processo de inclusão é a impossibilidade de estes responderem às necessidades dos alunos de forma satisfatória. Neste sentido, um estudo realizado por Cecil, Christopher e William (2002) revelou que a maioria dos professores que deixaram a área de educação especial o fizeram por se sentirem desprezados, sobrecarregados pelas necessidades dos estudantes, responsabilidades do trabalho e sensação geral de terem cada vez menos poder. De acordo com estes autores, a taxa de atrito e de abandono da profissão é maior em professores de educação especial do que em professores do ensino regular.

Em relação às diferenças de género, Esteras, Chorot e Sandín (2014), no seu estudo composto por uma amostra de 171 docentes de ensino regular, que exercem em instituições públicas e privadas, sobre o papel dos fatores organizacionais, pessoais e sociodemográficos como preditores de burnout na docência, identificaram com diferenças estatisticamente significativas entre géneros nas dimensões despersonalização e falta de realização profissional. Neste caso, os homens revelaram um maior nível, comparativamente às mulheres.

Martins (2008) encontrou valores mais elevados nos homens na dimensão despersonalização, não existindo diferenças significativas nas restantes, contudo, foram também os homens que apresentaram maiores níveis de exaustão emocional e as mulheres níveis mais elevados 
de realização pessoal. $\mathrm{O}$ estudo realizado por Amorim (2007), sobre a síndrome de burnout em professores portugueses, não apresentou resultados com diferenças significativas entre homens e mulheres nas várias dimensões. Contrariamente, a investigação realizada por Santos (2011), com uma amostra de 120 professores de ensino regular, indica a existência de valores mais elevados no género feminino, com resultados estatisticamente significativos. Há, assim, evidências de que são as mulheres que experienciam maiores níveis de stresse e burnout, contudo, não existe ainda um consenso afirmativo (Seibt, Spitzer, Druschke, Sceuch \& Hins, 2013).

Boujut, Dean, Grouselle \& Cappe (2016), concluíram que os professores que trabalham com crianças com deficiência não apresentam níveis mais elevados de burnout que os professores do ensino regular, facto que pode estar associado a estratégias de coping desenvolvidas na formação específica.

$\mathrm{Na}$ comparação de género, os estudos anteriores não reúnem consenso. Alguns estudos apontam para resultados mais elevados para os homens (Martins, 2008; Esteras, Charot \& Salin, 2014) e outros para as mulheres (Norlund, Reuterwall, Hoog, Lindah, Janler $\&$ Birgander, 2010). No entanto, estudos desenvolvidos em Portugal (Baptista, 2013; Carlotto, 2011) confirmam que as professoras de educação especial apresentam níveis mais elevados de stresse e burnout que os homens.

\section{Método}

O presente estudo de natureza correlacional tem como objetivo comparar os níveis de burnout, em professores de ensino regular e educação especial que trabalham com alunos com Necessidades Educativas Especiais, em escolas públicas portuguesas.

\section{Participantes}

A amostra é constituída por 7509 docentes, dos quais 7058 (94\%) são professores de ensino regular e 451 $(6 \%)$ de educação especial (média $=1,06 ; \mathrm{DP}=.238)$. Dos docentes de ensino regular, 5451 (77.2\%) participantes são do género feminino e 1607 (22.8\%) de género masculino (média $=1,23 ; \mathrm{DP}=.419$ ) e a sua média de idades encontra-se nos 50 anos ( $\mathrm{DP}=7.485$ ). Em relação ao grupo de docentes de educação especial, este é composto por 406 participantes de género feminino e 45 de género masculino (média $=1,10$; DP $=.300)$ e a sua média de idades encontra-se nos 49 $\operatorname{anos}(\mathrm{DP}=7.129)$.

\section{Instrumentos}

De forma a analisar os níveis de burnout manifestados nos docentes, recorreu-se ao Maslasch Burnout Inventory (MBI), aferido à população portuguesa por Martins (2008) através de uma amostra constituída por 450 professores de diferentes níveis de ensino. O MBI é um questionário composto por 22 afirmações, cuja forma de resposta é em escala tipo likert, variando entre 1 (Nunca) a 7 (Todos os dias). É solicitado aos participantes que assinalem uma resposta, tendo como referência os últimos três meses de atividade profissional. Este instrumento avalia três fatores: exaustão emocional, composta por 9 itens (exemplo de item: "Sinto-me cansado quando me levanto para ir trabalhar); despersonalização, da qual fazer parte 5 itens (exemplo de item: "Sinto-me preocupado porque este trabalho me deixa mais frio emocionalmente"; e realização pessoal, na qual se inserem 8 itens (exemplo de item: "Sinto que consigo fazer coisas úteis no meu trabalho").

\section{Procedimento}

O inventário de burnout (versão de Martins, 2008) foi impresso em papel e testou-se a sua usabilidade e funcionalidade em formato eletrónico na plataforma Limesurvey (versão 2.05) com alunos de mestrado em Educação, Educação Especial e Psicologia $(\mathrm{N}=20)$. O questionário foi submetido ao Conselho Científico da Escola de Ciências Sociais da Universidade de Évora (CC-ECS-UE) e aprovado pelo CC-ECS-UE em 23 de novembro de 2015 e também apresentado para aprovação da Direção Geral de Educação, Monitorização de Inquéritos em Meio Escolar. De seguida, divulgamos o estudo através de todos os agrupamentos das escolas públicas portuguesas, fazendo uso da lista de contactos das escolas públicas disponibilizadas pelo Ministério da Educação. Cada agrupamento de escola recebeu uma solicitação de email para divulgar o link do questionário aos seus professores, que o preencheram e enviaram para um email especialmente criado até 30 de janeiro de 2017. Para criar uma base de dados, analisámos cada questionário recebido na plataforma Limesurvey e inserimo-lo numa base de dados para posterior análise no programa IBM SPSS - Statistics Data Document (versão 22).

\section{Resultados}

Realizou-se primeiramente uma análise de variância univariada, de forma a compreender quais as dimensões que apresentam diferenças entre os grupos (tabela 1), tendo-se verificado que são os professores de ensino regular que expressam um maior sentimento de realização pessoal e profissional mas também de despersonalização.

Tabela 1.

Comparação dos níveis de burnout em professores de ensino regular e educação especial

\begin{tabular}{cccc}
\hline & & Regular & Especial \\
\hline Dimensões & $p$ & Média & Média \\
\hline Exaustão emocional & .170 & 36.28 & 35.50 \\
Realização Pessoal e & .013 & 20.19 & 19.26 \\
Profissional. & & & \\
Despersonalização & .020 & 14.16 & 13.35 \\
\hline \multicolumn{3}{c}{ Diferença significativa em $p .<.005$}
\end{tabular}


Analisou-se também as diferenças de género para os diferentes contextos de ensino (tabela 2), tendo-se verificado que são as mulheres professoras de ensino regular que têm um maior nível de exaustão emocional enquanto os homens se sentem mais despersonalizados. Para o grupo de educação especial não foram encontradas diferenças significativas.

Tabela 2.

Comparação dos níveis de burnout em professores de ensino regular (ER) e educação especial (EE) em função do género

\begin{tabular}{|c|c|c|c|c|}
\hline & \multirow[t]{2}{*}{ Dimensões } & \multicolumn{2}{|r|}{ Fem. } & \multirow{2}{*}{$\begin{array}{l}\text { Masc. } \\
\text { Média }\end{array}$} \\
\hline & & $p$ & Média & \\
\hline & $\begin{array}{l}\text { Exaustão } \\
\text { emocional }\end{array}$ & .000 & 37.22 & 33.07 \\
\hline \multirow[t]{3}{*}{ ER } & $\begin{array}{l}\text { Realiz. Pessoal } \\
\text { e Profissional }\end{array}$ & .357 & 20.14 & 20.35 \\
\hline & $\begin{array}{c}\text { Despersonalizaç } \\
\text { ão }\end{array}$ & .000 & 13.80 & 15.37 \\
\hline & $\begin{array}{c}\text { Exaustão } \\
\text { emocional }\end{array}$ & .207 & 35.72 & 33.51 \\
\hline $\mathrm{EE}$ & $\begin{array}{c}\text { Realiz. Pessoal } \\
\text { e Profissional }\end{array}$ & .456 & 19.35 & 18.42 \\
\hline & $\begin{array}{c}\text { Despersonalizaç } \\
\text { ão }\end{array}$ & .100 & 13.17 & 14.84 \\
\hline
\end{tabular}

Diferença significativa em $p .<.005$

\section{Discussão e Conclusão}

Os resultados encontrados neste estudo comparam os níveis de burnout em professores de ensino regular e educação especial que trabalham com crianças e jovens com Necessidades Educativas Especiais. Emergem duas conclusões principais: primeiro, são os professores do ensino regular que apresentam níveis mais elevados de exaustão emocional e despersonalização, segundo são as professoras do ensino regular que se apresentam mais exaustas, enquanto os professores do ensino regular se manifestam mais despersonalizados.

Estes resultados evidenciam, por um lado, um maior nível de realização pessoal e profissional, no grupo de professores do ensino regular mas é também este grupo que apresenta níveis mais elevados de burnout, em termos de despersonalização e exaustão emocional, o que vai no sentido oposto aos resultados encontrados em estudos anteriores, como por exemplo, Boujut, Dean, Grouselle e Cappe (2016). Nos professores do ensino regular, no que se refere às diferenças de género, apesar de alguns autores (Seibt, Spitzer, Druschke, Sceuch \& Hins, 2013) referirem não existir ainda um consenso afirmativo, observou-se que, à semelhança de outros estudos (Martins, 2008; Esteras, Charot \& Sandin, 2014; Kuch, 2011), são as mulheres que estão mais exaustas emocionalmente enquanto os homens mais despersonalizados. Estes resultados podem ser interpretados em função do contexto social e político que se vive em Portugal em 2016-2017, decorrentes da crise económica vivida em Portugal com consequências ao nível da gestão e organização do trabalho nas escolas, para além do alargamento da escolaridade obrigatória a todos os alunos, incluindo todos os alunos com necessidades educativas especiais independentemente do nível de complexidade e intensidade do apoio necessário para os alunos.

Neste sentido e de acordo com a investigação neste campo, destacamos a necessidade de ter em conta um conjunto de medidas que apoiem os professores do ensino regular no processo de educação e ensino inclusivo dirigido a todos os alunos, nomeadamente:

- Reforçar o trabalho de equipa entre professores do ensino regular e professores de educação especial. Será necessário que a escola atribua mais tempo aos professores para este trabalho conjunto.

- Organização dos horários dos professores de modo a permitir o trabalho em par pedagógico de forma a garantir que os professores de educação especial possam estar em sala de aula, num trabalho de verdadeira parceria pedagógica com o professor da turma, tal como sugere a legislação em vigor, em prol de uma educação verdadeiramente inclusiva.

- Reforçar a formação em educação especial e estratégias pedagógicas de diferenciação junto dos professores do ensino regular, de modo a garantir que todos os professores terão uma preparação mínima para garantir a educação e ensino inclusivo para todos os alunos.

Estes resultados desafiam-nos a continuar a acompanhar a incidência dos níveis de burnout em professores portugueses em estudos futuros, combinando dados mais descritivos quer em termos de perceção do suporte social quer em termos quantitativos, como também qualitativos, uma vez que este se insere nas estratégias de coping utilizadas pelos docentes e o qual tem um efeito protetor e direto no burnout (Martins, 2008), quer em termos de consequências para a saúde e o absentismo dos professores.

A finalizar destacamos a importância que estes resultados devem ter no planeamento da saúde e bemestar do professor na escola. Como referimos, o burnout tem implicações na saúde e no desempenho profissional, como demonstrado em estudos prévios (Romão, 2012), pelo que consideramos fundamental desenvolver medidas de intervenção organizacional e profissional que apoiem o professor na minimização dos efeitos da exaustão emocional e despersonalização, através de programas de desenvolvimento profissional, formação contínua, grupos de apoio e programas para gerir stresse e burnout, promovendo o bem-estar e a saúde dos professores. 


\section{Referências}

Amorim, I. (2007). Nuevos Contextos de Intervención Psicológica en Educación, Salud y Calidad de Vida. International Jounal of Developmental and Educational Psichology, 1 (1).

Baptista, A. (2013). Stress e Burnout em Professores de Alunos com Necessidades Educativas Especiais. Dissertação de Mestrado. Universidade Católica Portuguesa.

Barasuol, E. (2004). Burnout Docente no Trabalho com a Inclusão de Alunos com Necessidades Educacionais. Dissertação de mestrado. Rio grande do sul.

Capitão, P. (2010). Concepções dos Professores face à Inclusão de Alunos com NEE no Ensino Regular. Dissertação de Mestrado. Escola Superior de Educação de Lisboa.

Carlotto, M. (2002). A Síndrome de Burnout e o Trabalho Docente. Psicologia em Estudo, 7 (1), 2129.

Carlotto, M. (2011). Síndrome de Burnout em Professores: Prevalência e Fatores Associados. Psicologia: Teoria e Pesquisa, 27 (4), 403-410.

Cecil, F., Christopher, B. \& William, N. (2002). Teacher Burnout In Special Education: The Causes and The Recommended Solutions. High School Journal, 86 (1).

Cruz, S. (2012). Alunos com Necessidades Educativas Especiais: Dificuldades sentidas pelos professores de educação especial. Dissertação de Mestrado. Lisboa: Escola Superior de Educação Almeida Garrett.

Gomes, C. (2010). O lugar do sujeito na inclusão escolar: percalços e fracassos nas relações de subjetivação. Tese de Doutoramento. Pontifícia Universidade Católica de Campinas - PUCCAMP.

Maslach, C., Schaufeli, W. \& Leiter, M. (2001). Job burnout. Annual Review Psychology, 52, 97-422.

Martins, J. (2008). Burnout na profissão docente. Dissertação de Mestrado. Porto: Faculdade de Psicologia e Educação.

Morais, M. (2011). Burnout em docentes envolvidos na inclusão de alunos com Necessidades Educacionais Especiais na rede pública. Brasília: Instituto de Psicologia

Norlund, S., Reuterwall, C., Hoog, J., Lindah, B., Janler, U. \& Birgander, L. (2010). Burnout, working conditions and gender- results from the northern Sweden Monica STUDY. BMC Public Health. doi: 10.1186/1471-2458-10-326.

Pinto, N. \& Morgado, J. (2012). Atitudes de pais e professores perante a inclusão. In L. Mata, F. Peixoto, J. Morgado, J. C. Silva \& V. Monteiro (Eds.), Actas do $12 .^{\circ}$ Colóquio Internacional de Psicologia e Educação: Educação, aprendizagem e desenvolvimento: Olhares contemporâneos através da investigação e da prática (pp. 471-491). Lisboa: ISPA - Instituto Universitário.

Romão, C. (2012). A cooperação entre educadores de infância e docentes de educação especial na inclusão de crianças com NEE e as crianças índigo? Qual o seu lugar?, Universidade Fernando Pessoa, Porto.

Santos, S. (2011). Burnout e diferenças de género em professores. Dissertação de Mestrado. Lisboa: Universidade Lusófona de Humanidades e Tecnologias.

Seibt, R., Spitzer, S., Druschke, D., Scheuch, K. \& Hinz, A. (2013). Predictors of mental health in female teachers. Int J Occup Med Environ Health, 26 (6), 556-869

Silva, M. (2011). Educação Inclusiva: um novo paradigma de Escola. Revista Lusófona de Educação, $19,119-134$ 